\title{
Real-Time Cloud-Based Health Tracking and Monitoring System in Designed Boundary for Cardiology Patients
}

\author{
Aamir Shahzad $\mathbb{D}^{1},{ }^{1}$ Yang Sun Lee, ${ }^{2}$ Malrey Lee, ${ }^{3}$ Young-Gab Kim $\mathbb{D}^{1},{ }^{1}$ and Naixue Xiong $\mathbb{D}^{4}$ \\ ${ }^{1}$ Department of Computer and Information Security, Sejong University, 209 Neungdong-ro, Gwangjin-gu, \\ Seoul 05006, Republic of Korea \\ ${ }^{2}$ Division of Convergence Computer \& Media, Mokwon University, Deajeon, Republic of Korea \\ ${ }^{3}$ Advanced Image and Information Technology, School of Electronics \& Information Engineering, Chonbuk National University, \\ 664-14 1Ga, Deokjin-Dong, Jeonju 561-756, Republic of Korea \\ ${ }^{4}$ Department of Mathematics and Computer Science, Northeastern State University, 611 N. Grand Ave No. 323, Webb Center, \\ Tahlequah, OK 74464, USA
}

Correspondence should be addressed to Young-Gab Kim; alwaysgabi@sejong.ac.kr and Naixue Xiong; xiongnaixue@gmail.com Received 24 November 2017; Accepted 28 February 2018; Published 21 May 2018

Academic Editor: Ka L. Man

Copyright ( 2018 Aamir Shahzad et al. This is an open access article distributed under the Creative Commons Attribution License, which permits unrestricted use, distribution, and reproduction in any medium, provided the original work is properly cited.

\begin{abstract}
Telemonitoring is not a new term, in information technology (IT), which has been employed to remotely monitor the health of patients that are located not in common places, such hospitals or medical centers. For that, wearable medical sensors, such as electrocardiography sensors, blood pressure sensors, and glucometer, have commonly been used to make possible to acquire the real-time information from the remotely located patients; therefore, the medical information is further carried, via the Internet, to perform medical diagnosis and the corresponding treatments. Like in other IT sectors, there has been tremendous progress accounted in medical sectors (and in telemonitoring systems) that changes the human life protection against several chronic diseases, and the patient's medical information can be accessed wirelessly via Wi-Fi and cellular systems. Further, with the advents of cloud computing technology, medical systems are now more efficient and scalable in processing, such as storage and access, the medical information with minimal development costs. This study is also a piece of enhancement made to track and monitor the real-time medical information, bounded in authorized area, through the modeling of private cloud computing. The private cloud-based environment is designed, for patient health monitoring called bounded telemonitoring system, to acquire the real-time medical information of patients that resided in the boundary, inside medical wards and outside medical wards, of the medical center. A new wireless sensor network scenario is designed and modeled to keep or monitor the patients' health information whole day, 24 hours. This research is a new secured sight towards medical information access and gives directions for future developments in the medical systems.
\end{abstract}

\section{Introduction}

In medical sectors, employing of pervasive medical devices and their connectivity with the advance networks or/and the Internet brought the new visions for human medical diagnoses, treatments and monitoring, wireless body area network (WBAN), and remote monitoring of patients' health. The pervasive devices or medical sensors are connected with the specific parts of patients' bodies, to measure the acquired medical information such as blood pressure, sugar level, heart rates, and other medical signals, and the observed medical information will be transmitted to the medical assistance or medical advisor, through the connectivity of wireless media including cellular networks, where the received medical information will be examined for further diagnosis. Automated medical analytic tools, such as electrocardiogram analyzers, are also available for medical information analyses in real-time manners and are accounted as a part of the telemonitoring system [1]. As telemonitoring systems are not the new technological solutions in the monitoring of patients' health, several medical healthcare systems [2] have been deployed to monitor the indoor 
or/and remotely located patients' health status to overcome the emergency cases and to fight against and diagnose the critical diseases before they become worst. To be more enhanced, the technologies called cloud computing systems are efficient and scalable solutions for existing networks and have been playing tremendous roles in healthcare systems in terms of information monitoring, acquisition, and storage. Through employing and deploying of public cloud computing technology for healthcare, the overall processing of healthcare systems is much efficient and easy to manage. It means that the hospital can just use the services of public cloud to underpin the continuum healthcare and also can manage the administration and other required IT requirements that have the potential to retrieve the real-time information of patients without any delay, truly synchronized and securely shared among the systems (and users), and scalable in cases of workload, and the information is always be accessible when required. In inclusion, through employing of cloud computing infrastructure, a health organization is able to manage its overall organizational structure, as a solution emerges with the coherent-optimal healthcare system. Further, in cloud computing, the public cloud is efficient in the monitoring of information and managing of services, for pervasive healthcare systems and be deemed appropriate for the issue of scalability and security. As the retrieving of medical information has great value and requires to be secured during transmission over the Internet, therefore several medical organizations have not been attaining upon the public cloud platforms, due to the security issues and to gain the high-security level during information exchanges. In short, public cloud services are efficient and reliable, but they still encompass potential vulnerabilities because they are called publicly open for all. To resolve the issues of security, the private cloud computing is a significant and trusted solution for medical information exchanges, with the satisfactions of information confidentiality and authorized access; moreover, medical organizations can also leverage their other important resources [2-5]. Figure 1 illustrates the typical structure of cloud computing for healthcare systems.

Eucalyptus, called "Elastic Utility Computing Architecture for Linking Your Programs to Useful Systems," mainly deploys for private and hybrid cloud computing services and is an open software used to implement infrastructure as a service (IaaS), network as a service, and storage as a service, and these services are made available for end-users by consolidating with other existing infrastructures in cloud computing environment [6, 7]. In [8], ECG analyses were performed by hosting application (or analysis software), as software-as-a-service (SaaS) and as a web service, in public cloud computing environment. In cloud computing environment, the platform-as-a-service ( $\mathrm{PaaS}$ ) layer used three main modules, such as "container scaling manager or tomcat container, workflow engine as service platform, and Aneka application platform," to manage the processing of hosted software and to fulfill the demands/requests from users. Therefore, upon user requests, container scaling manager scales the number of containers to process the user queries toward workflow engine that controls the overall processing and jobs assigned to the medical software workflow and further processes them to PaaS middleware called Aneka. Aneka is an innovated technology based on Microsoft.NET platform, an application development platform (for distributive applications) which provides run-time development scenarios and application programmable interfaces to develop customized applications, on either private or public cloud computing platform [9]. Aneka software, a development platform, provides several services in the development phases of distributive applications, for example, defines useful logic for distributive applications. Moreover, it is a generic infrastructure that manages the processing of distributive applications and is a consolidation platform to work with other existing infrastructures (e.g., "Amazon EC2" as part of cloud computing [10]. Each workflow engine was hosted, as a part, inside a tomcat container and would be increased in numbers based on the work demands from the users. Sooner, the user jobs are being collected by the workflow engine and processed to the Aneka, where each job resided in the waiting queue is triggered depending on the availability of resources. Therefore, by using of the masterworker scenario, Aneka manages the upcoming communication between the IaaS that runs Aneka master service and Aneka workers, installed in VM at PaaS. Furthermore, waiting-request scheduling at the workflow and the average user-requests handled by the container in specific session are performed in run-time through cloud load balancing $[8,9]$.

As concluded, the most efficient way to manage an organization's dedicated resources, especially from the encounter of security, and keep their information internally as part of organizational regulations without enrolments of external entities (e.g., outside of the organization), is private computing platform. Cloud computing provides and manages an elastic pool of resources based upon requirements by the end-users' perspectives; private cloud computing employs in case the resources that are designated for a single organization and public cloud computing in the case of multiply organizations shared the resource pool. The difference between the public and private computing is very little; for example, large scaling of Internet services and applications are mainly managed through employing of public cloud and the virtualization technology is utilized through private cloud computing; but at the same time, they have some very common characteristics, such as pool of resources, independent access, resource elasticity and management, and resource metering [6].

For the life demands and to improve and to fulfill the medical requirements of human lives, the notable changes have been accounted in medical sectors that are significant not only for the indoor patients but also for the remotehome residential patients. The use of wireless sensor devices and sensor networks made this possible to provide the medical cares and monitor the facilities for the remote patients. The continuous monitoring of remote (located) patients' status (i.e., blood pressure, heart rates, sugar level, and others) is significant in order to detect the critical diseases before the emergency conditions happening and also provides various medical services for the caregivers to manage the risk cases of patients and their cognitive disorders, while happening 


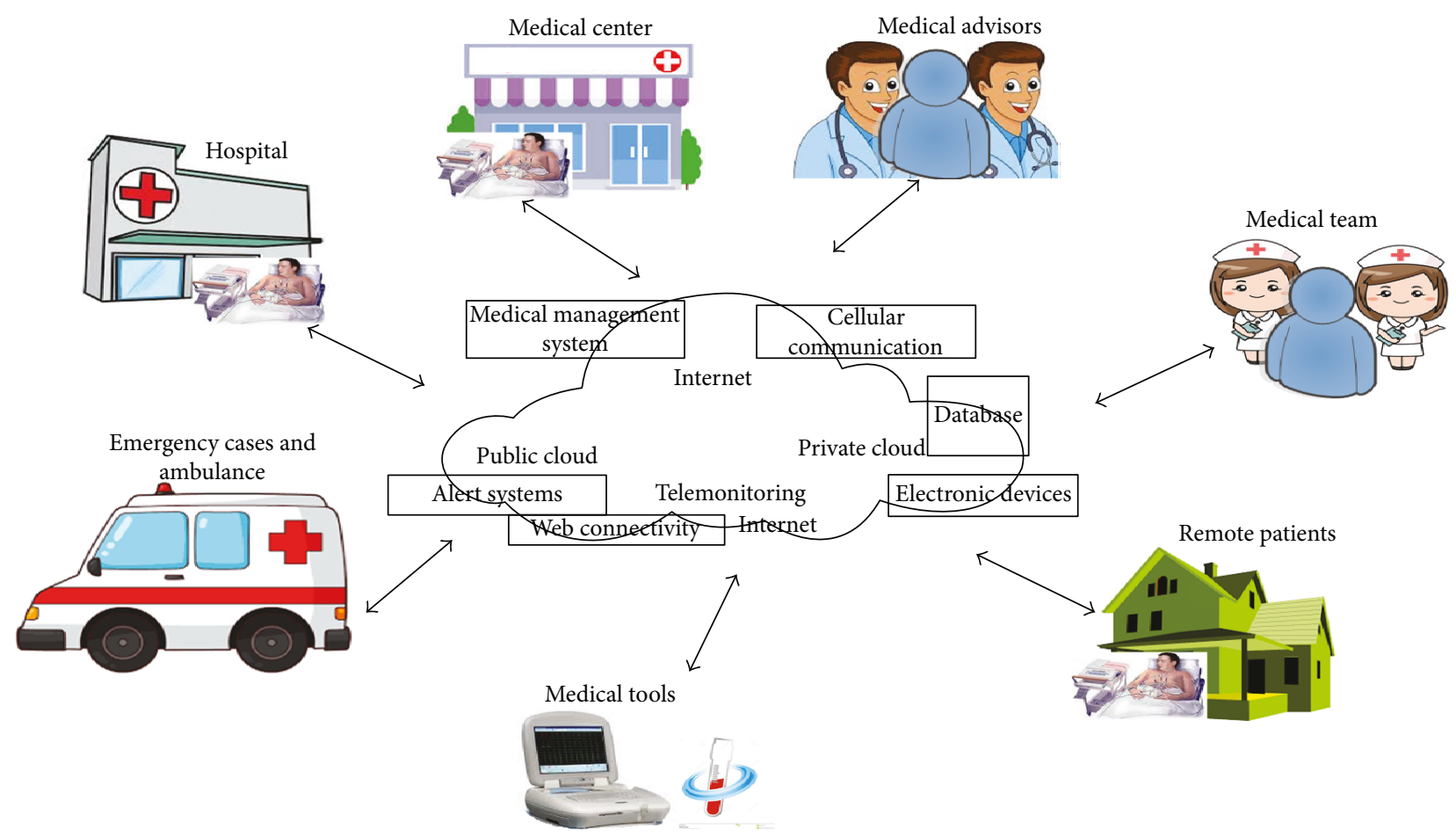

FIGURE 1: Typical cloud computing structure for healthcare systems.

$[11,12]$. Usually, the medical sensor devices are embedded or placed on specific parts of the patient where the medical rates (or information) are required to measure, and the observed information is further transmitted wirelessly, monitored, and stored at the designated controller site (or computer machine) where the authorized persons (or authorized medical advisor) are situated for information analyzing and diagnosis purposes [12]. In last decades, a number of researches have been conducted for the development of advance context-aware medical applications, by a combination of computer networks, cellular networks (e.g., 2G, GRPS, and $3 G)$, and wireless sensor networks. On the other side, with the great development of unobtrusive sensor devices, Bluetooth devices, and radio frequency identification- (RFID-) based sensor devices, for medical systems, their automated configurations with wireless networks make it possible to process the patients' acquisition information, from anywhere, from anyplace. These enhancements made are accounted as tremendous changes in medical systems and in reducing of critical sudden conditions of patients during an emergency and in reducing the effects of diseases, as the diseases are diagnosed and handled before going to be critical. The remote monitoring of patients' health or telemonitoring systems via sensor devices and the use of wireless sensor networks play an important role in making a coordination between the patients and medical advisor and also between medical sensor devices and other parts of the telemonitoring system [11-15].

Upon the requirements, which commonly specified by health organizations and the health center (or hospital) in our case, building a private healthcare system also mainly targets the security obstacles as its main goal. The idea to deploy a private cloud platform, for the healthcare system, is significant in ways to reduce the cost and usage of the scalable network and the available resources $[6,8,16]$. In this study, through the proposed solution to access and monitor the medical information from patients in cases of the inward system and outward system, private cloud computing platform is designed by employing of virtualization technology in Microsoft.Net platform which allows to design and employ the private cloud computing platform, for medical information monitoring, analyzing; and automated classification, as infrastructure-as-a-service. In fact, the main goal of this study is to access and monitor the real-time information of cardio-patients through the usage of built-in ECG belt having embedded sensor and ECG device where required. Therefore, the private cloud platform is modeled only that would easy to make integration with other modules of the proposed system such as cellular module, ECG acquisition module, Web application, and security and secure logon.

The rest of this research paper is organized as follows. Detailed literature is conducted in Section 2, and Section 3 describes the proposed system design and modeling, including private cloud design, inward monitoring, and outward monitoring. Study results are conducted in Section 3, and discussions are made. At the end, in Section 4, overall work study is concluded and future work is described.

\section{Literature Survey}

As the enhancements are made in telecommunication sectors, the telemedicine systems are discovered and as the passage of time, these systems have also been updated with the new technologies (i.e., telemonitoring systems) which are 
significant in case of disease diagnosis and patient medication and remote monitoring of patients and treatments; by this way, the patients can be monitored from the places (e.g., medical centers and homes) where they are situated, over the cellular platform. For that, depending upon the demands, a new system is required that not only provides healthcare services inside the hospitals or healthcare centers but also provides efficient healthcare facility to remotely located patients. Therefore, the telemonitoring system is the best solution which provides medical monitoring facilities for the distance located patients and for the medical advisors. Especially, in the situation to fight against cardiology or arrhythmia that will always happen suddenly_if it happened-therefore, a possibility to monitor it is through remote networked portable ECG telemonitoring system [17-20]. In case of emergency, ECG-measured information is observed and simultaneously transmitted through cellular transmission, satellite transmission, and other computer wired/wireless networks to the control center or server, where the observed information will further be assessed for diagnosis and storage purposes $[19,20]$. Usually, in telemonitoring systems, the carried information is saved into two storage modes: real-time mode, which stored information in real-time manners as its monitoring from ECG devices and in store-and-forward mode, where the carried (monitoring) information is latterly stored, after the specific session, and used for health analysis purposes [21].

The sensor devices and their utilization have prominent placed, in order of patient's medical treatments, in hospitals or/and healthcare centers. The sensing technology, employed in hospitals, is not very old (i.e., ECG or EKG) but has been changed as the passage of time corresponding to the technology enhancements and medical system requirements. Nowadays, various medical sensors have been used in hospitals in order to keep the physiological and physical status of patients, patient health information is tracked in real-time manners and diagnosis, and corresponding treatments are applied. The transducer devices are mainly used with medical sensors that detect the electric, genetic, and other signals, with the initialization of physiological signals combined with signal processing algorithms that predict the future status or behaviors of patient's health. Besides this, several meaningful applications, such as "monitoring in mass-casualty disasters, vital sign monitoring in hospitals, at-home and mobile aging, assistance with motor and sensory decline, and large-scale on-field medical and behavioral studies," relevant with healthcare systems were reviewed and major security challenges were also deemed that might be existing in wireless sensor networks (WSNs) to ensure the future privacy of medical sensitive information [22-24].

In a study [19], a new RFID tag called "miTag (medical information tag)" was introduced and employed during emergency cases or health disaster situations. This was a cost-effective solution, by employing tag, that provided inclusive information by tracking and monitoring the medical conditions of patients in each step (or state) of disaster, such as from the place where the disaster happened to the ambulance and from the ambulance to the hospital. Whereas, several controllers were designated and were able to view the overall information while connected to wide area network
(WAN), to manage the number of patients in emergency units. The design of miTag was deemed highly scalable and extensive that can be used with a variant of communication systems, that is, GPS, and able to sense the patient status such as blood pressure rate, body temperature, and ECG signals. The miTag device was used and supported to transmit information in two-way directions. In the disaster scenarios, several major requirements, such as scalable usage of sensors and networks, reliable and efficient transmission ways, and costless and efficient hardware devices, have to be considered in order to track the remotely located patients' information. For that, few commercial devices are available, but most of them have a number of limitations, such as network setup and configuration issues; tracking is only limited to persons unable to identify the disaster locations, a poor installation, and integration with other systems, and so on, which therefore significantly affects the overall transmission $[12,16,25,26]$.

2.1. Case Study: ECG Telemonitoring System via Cellular Platform. In Figure 2, a telemonitoring system is proposed, which monitors the cardiology patient's information through connecting with ECG (electrocardiogram) device, in realtime mode and store-and-forward mode which encompassed a Holter and a controller (or server). While required, patient information is delivered via MMS (multimedia messaging service) through GPRS (general packet radio service)/GSM (global system for mobile communication) networks. Holter is a patient-worn unit where patients are situated, and ECG devices are connected with them and provided GPS (global positioning system) information which would be helpful in cases of emergency. Holter is also responsible for patients' ECG information, its storage, and assessment. In the realtime mode, patients' abnormal heartbeats rates are measured, to make be efficient, in order to measure the accurate heart rates. To do this, an algorithm is deployed that made the classifications, on measurements, of heart rates which would be significant in finding the abnormal heart rates, and based on the measured rates, the corresponding medical advisor would be concerned through MMS, in real-time manners. MMS is a new technology and developed under the 3GPP (third generation partnership project), which made it possible to transmit the multimedia messages (e.g., text, audio, and video) that varied in sizes. As the information is measured from ECG device which is directly attached to the patient, and the measured information further assessed (or classified), with the classification algorithm, resulted in the abnormal heart rates, then at the same time, this information is transmitted to the server in the form of MMS, with the embedded information of Holter-GPS. GSM/GPRS networks are utilized for MMS, and further, the patient's monitored information is transmitted to the MMS center to the server via TCP/IPs. Upon received information, server stored this patient (monitored) information and autostreamed this information to the medical advisors. Authorized medical advisor analyzes the received information, generates the corresponding feedback, and is authorized to send MMS back to the server to keep connected with the patient for the prospective information. In server end, GIS (geographic information system) software is installed that is used to locate the patients 


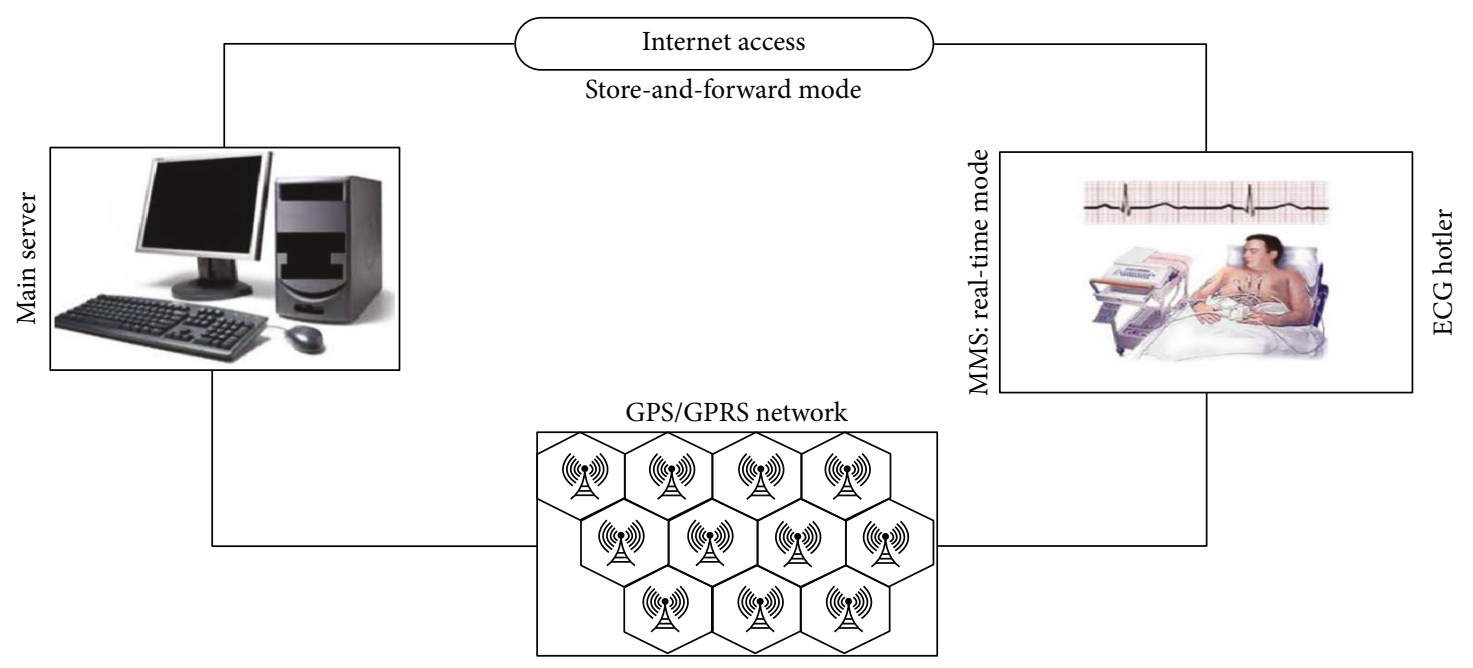

FIgURE 2: Telemonitoring system via cellular access [21].

in emergency cases; as the delivered MMS was packed with GPS information, therefore, this information is further used to locate the patient's location (i.e., Holter location). At both sides, in either MMS sent from the patient or sent from the medical advisor, the communication always carried in realtime senses. Real-time mode is more reliable, as the MMS (e.g., patient abnormal heartbeat rates) is transmitted, stored, and analyzed in minimal time, or without any time delay. While in case of store-and-forward mode, the Holter measured and stored the patient ECG data continuously up to 48 hours and sent the monitored information to the server for storage and future analysis purposes. In this case, information is carried through the internet, in the presence of wired networks and TCP/IPs [21].

As analyzed from above, considerable changes have been accounted in medical systems due to the gained and deployed wireless sensor technologies. The existing works $[18,19]$ were also conducted on to monitor the patient's status or conditions by means of various detection approaches such as fall detection, gesture and posture detections, and GPSbased tracking for patients and through several medical sensors and RFID tags that have been very commonly employed in telemonitoring systems [21]. The telemonitoring systems have been playing very important roles in reducing the effects of sudden critical diseases (i.e., arrhythmia causes) and give future trends for pervasive healthcare systems. With those systems, several benefits will be achieved and with these benefits, the patients should able to track and monitor the risky situations during emergency. Furthermore, the children and old people, who might have to suffer from cognitive and chronic illness (and other physical disorders), can also be attained in order to reduce the effects of their diseases, with safer, happier, and more independent lives [19, 21, 24]. To make a new extension as a part in healthcare systems or telemonitoring systems, the technology called cloud computing is required in which cardiology patients will be undertaken and monitored through the RIFD-based medical tags that are considered to be implanted in patients' bodies (i.e., over their hearts) and real-time information, for example, heart normal and abnormal rates, will be captured, monitored, analyzed, stored, and viewed by the authorized medical advisors and also will be viewed by the family member of the patient under security authorization rules.

As a consequence, to gain the high efficiency, scalability, and security, with the overall minimal consumption of cost, most of the information technology- (IT-) based systems, such as educational, industrial, transportation, and medical systems, are now connected and communicating through cloud computing platform which minimizes the difficulties and complexities of end-user workloads, without purchasing of any expensive software or hardware. Similarly, there is also a new trend of communication, also called ambient intelligence (AMI), based on ubiquitous communication platform, a cloud computing environment that provided interaction between the end-users and various electronic or/and sensor devices over wireless sensor networks, a context-ware design which fulfill users' demands based upon their technological requirements [27].

In the proposed Cloud-IO design, three important services, telemonitoring, location identification, and communication service, were taken into account, which modeled in the way to be consolidated with each other and should be functional over the cloud computing environment.

(i) In telemonitoring service, the required information was gathered according to the situation and from users or patients, in premises of deployed wireless network, through the connectivity of various sensors, such as medical sensors and biometric sensor devices $[27,28]$.

(ii) A service, called location identification, was also accounted as a part of Cloud-IO, which provided the real-time identification of the cloud users and other network entities through estimating of their positions by deploying of location estimation algorithms. Those algorithms were efficient in managing of alarms and other indications acquired from cloud users and from configured system entities. Implementing the position estimation algorithms, such 
as more advances with the uses of frequency difference of arrival (FDOA) and time difference of arrival (TDFA) [29], that compute more accuracy compared with existing and are adequate in computations both for indoor and outdoor location estimations $[27,30]$.

(iii) In communication service, the text and voice messages were allowed to transfer over the designed cloud platform, through the employment of various wireless sensor devices, to fulfill the desired, overall communication goals, together with the microcontrollers as well as routing schemes and queuing managing algorithms are acquired for implementation [27].

\section{System Design and Modeling}

In information technology (IT) sectors, cloud computing is an efficient, scalable, and costless way to employ and deploy the organization's resources that include software, platforms, and infrastructures. A number of organizations have been employing the cloud computing platform to fulfill their organizational demands, industrial, and other requirements. In case an organization wants to keep its resources well and secured in its data center, away from the others or from external entities, the best solution that has been provided by cloud technology is private cloud computing by using the concepts of visualization [6]. In private cloud computing, the term visualization has a real meaning to deploy, employ, and manage the required organizational resources or applications running, inside the virtual environment, in virtual machines (VMs), rather install these applications in distinct hardware, in an organization, that consume high capital and management cost.

As explained above, private cloud has a special meaning for several information technology (IT) applications and services, including telemonitoring systems. Therefore, in the proposed study, a private cloud computing platform, using Microsoft.Net platform and System Center 2012, is considered and deployed as an efficient and cheaper way to access and measure the continuous real-time medical status or information of patients that are located in premises of hospital, such as inside the medical ward and outside the medical wards or in specified boundary of a hospital deemed in our study cases. This solution, System Center 2012, has been a commonly used designed platform for visualization, supporting three hypervisors: Microsoft Hyper-V, VMware ESX/ ESXi, and Citrix XenServer, and provides virtual machines on demands from users (e.g., company partners) [6], and is efficient in its services like supporting various applications (i.e., software and hardware), as well as monitoring, updating, and distributing among system entities. Figure 3 illustrates the proposed study design and its main components.

3.1. Private Cloud Computing Design and Modeling. For accessing and monitoring the medical information of heart patients residing in the hospital premises, private computing platform is built up by employing of System Center 2012 .
This system mainly encompasses few paradigms that provide the private cloud computing values for a new system development with the specified workloads and designated to the platform entities: (1) An administer entity is designated for building the cloud and perform updating process for applications that were deployed by IT experts. (2) A developer entity is designated for building the virtual environment by virtual machine (VM) concept and also authorized to provide approving process of the VM. (3) The cloud employing applications are designed and deployed by the IT experts, and (4) during an application running in private cloud computing, the system center is designated, correspondingly, for problem detection and fixing. In Figure 4, a proposed study about private cloud computing and design is illustrated with the employing of System Center 2012.

3.1.1. Private Cloud: Virtual Machine Manager. For private cloud computing, virtual machine manager (VMM) is a building block and the main management tool for managing the organizational data center. It provides the elementary services that are used in building and managing of clouds and makes the virtualization environment through virtual machines and concepts, in which the virtual cloud hosts, with resources (e.g., storage and cloud networking), are configured and managed through VMM, as part of private cloud computing design and its services. System Center 2012 provides services, such as server management service, console service, database or storage service, library service, command shell service, and security and scalability service, to deploy the virtual machine manager for efficient private cloud computing. In our case, the main four virtual machines are created corresponding to the medical wards, meaning that there are four medical wards considered, and each encompasses only ten patients for real-time medical information monitoring, and an API was installed for accessing and monitoring of the patients, while they are outside of their medical wards in a hospital area, which may consider as virtual machines, as part of private cloud computing. To do this, wireless sensor network has modeled in which a number of sensors are configured in hospital premises that are sufficient to access and monitor the patients' medical information, through employing of ECG belt and also their positions, in the boundary of the hospital. However, the proposed design inelasticity would be extendable with new sensors, if required.

3.1.2. Private Cloud: Application Controller. Application controller provides managing of the facilities, for private cloud platform, in order to manage the deployed applications, such as ECG information acquisition and storage, ECG classification algorithm, alarm indications, and API for ECG belt, and services for information access and monitoring, respectively. An integrated self-service web portal is also provided, which allows the users to make a request directed towards the designed private cloud, to deploy, configure, and manage the created VMs (e.g., a total of six virtual machines in our study cases) and other required cloud services. 


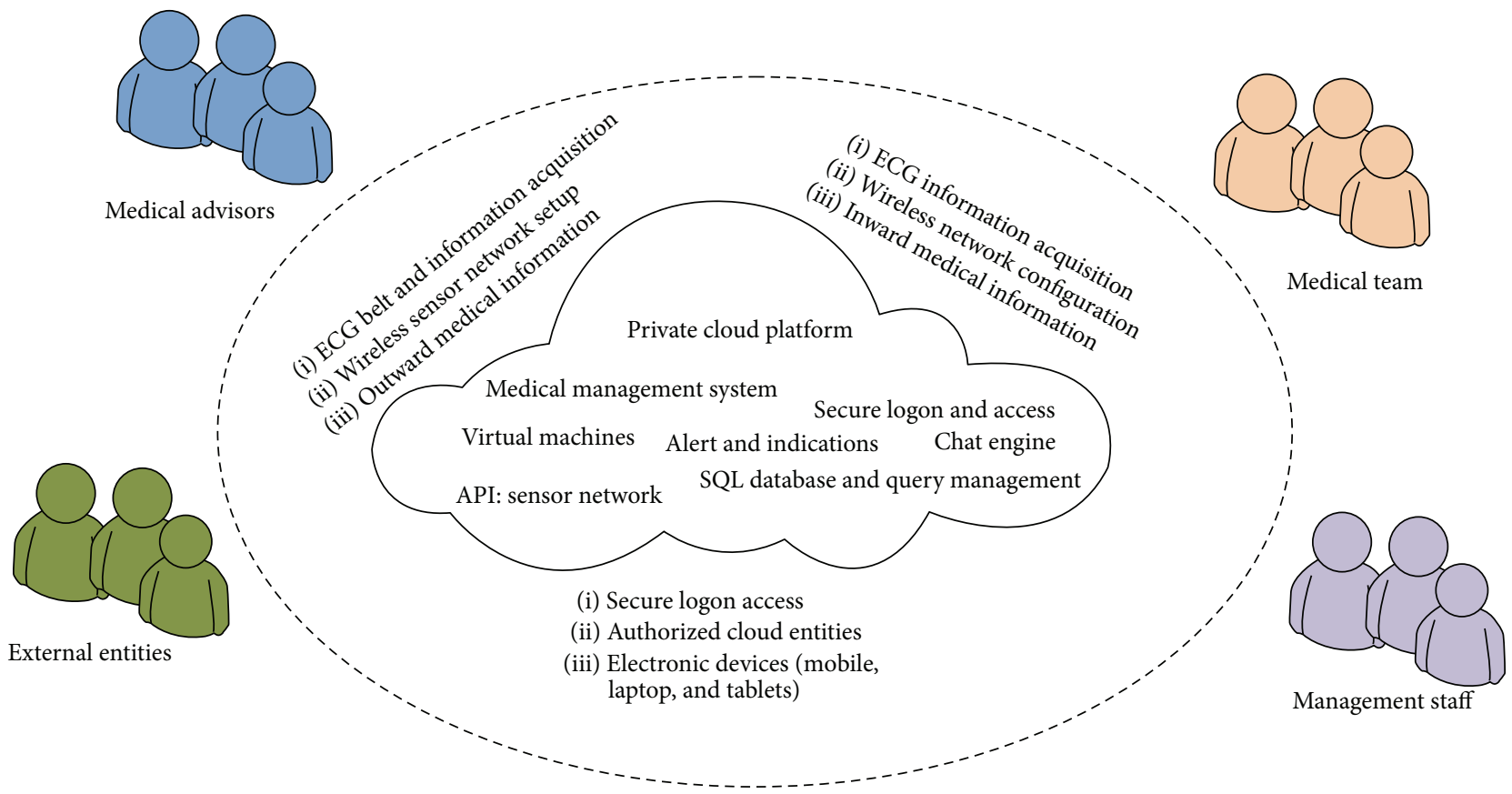

Figure 3: Proposed study design and components.

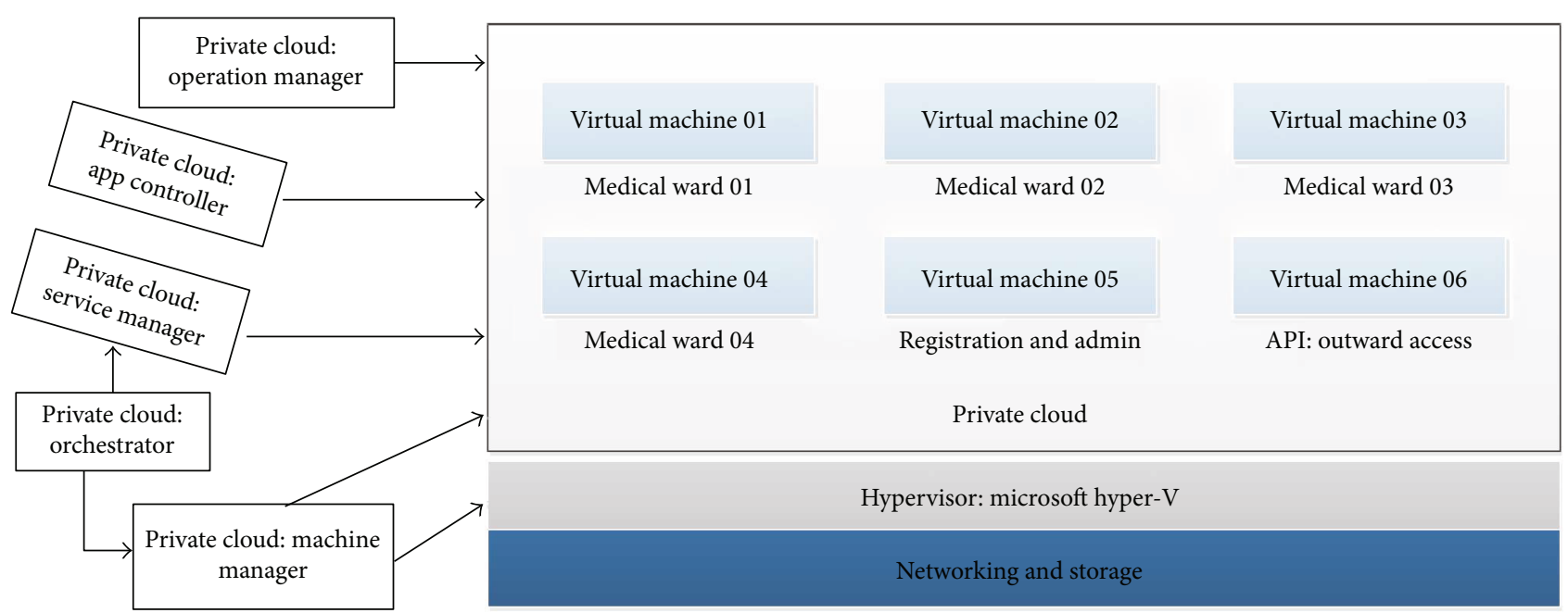

Figure 4: Proposed study: private cloud design.

3.1.3. Private Cloud: Service Manager. The service manager is an integrated, self-service portal and an automated management, as a part of private cloud computing, a platform for various services provided. For example, a new process is defined that has to manage a private computing environment, and before processing, permission criteria have to be fulfilled and should be possible with the support of service management. In our case, the external entities such as the patient's friends or family members are allowed to monitor their patients, residing in the hospital, the medical status, and other indications that will be given from the hospital management, through a secure login to the private cloud system.
3.1.4. Private Cloud: Orchestrator. In a private computing environment, an orchestrator is a solution, supported by data center, in order to manage the workflows, make interactions between employed management tools, and provide automated facilities for the users to build, implement, and monitor the resources in their own ways.

3.1.5. Private Cloud: Operation Manager. An operation manager is one of the important aspects of private cloud computing, which supports in order to monitor the running cloud virtual machines, applications, and services and perform the triggers against the issues while these happened and detected in a cloud environment. 
3.2. Databases. In this study, the database is one of the important modules which keeps a record of information that are retrieved from patients through the ECG device connectivity. Database tool, called MySQL, is employed to deploy and to keep the records of each patient medical information by means of identifier that makes a distinction between each patient's captured information in his/her identity. Each time information is transmitting, in real time manners; store in the form of table's records that will further retrieve based on the queries will be requested or required by the medical individuals.

3.3. Mobile Application Design and Software. In this study, a mobile application is the most important and one of the new developments modeled to retrieve the real-time information from medical devices attached to measure the patient cardioinformation. Therefore, for this purpose, medical application is designed and modeled, which will be installed in the authorized cellular device, with the registration of a user name and security password from the cloud, which makes the automated connectivity with the external medical devices (such as ECG devices) through Bluetooth and Wi-Fi accesses. Upon logging on to the cloud, the application is installed in a cellular device and the ECG information will be viewed only in real-time manners. The other electronic devices, such as laptops and tablets, can also be used to visualize the medical information, in real-time manners, prior to register via the cloud. Among other, the main goal of this study is to get measurements from the connected ECG devices and to generate corresponding reports, within the private cloud-computing environment, which means that overall medical system access is only limited and accessible within the medical center frontier. To do this, the wireless sensor network is designed and networked within the premises of a medical center; however, the coverage that attained through signal strength covered most parts of the medical center.

In this study, permanent caregivers are the individuals, medical advisors, and medical staff, which are the main entities attached to patients' care while they are in the medical center for the short and long medical treatments. Therefore, through the cellular medical application, the detailed access is provided; the access might be limited according to the designated patients. It means that few patients are designated for one doctor and 2-3 persons from the medical staff; the cellular application provides detailed real-time information of these patients only, which would be viewed on cellular device and the detailed report viewed in the table includes overall information about the existing and current medical information of patients. As the current medical system designed is also mainly focused to employ a potential security solution against the adversaries or unauthorized individuals that might reside inside the medical center boundary or intercepting from outside network. In both cases, the security has to be managed to avoid the inside/outside attacking issues, as the medical information is always somehow critical to protect. Therefore, shorter access for few days or based on patient treatment session, authorized access will be provided to patient's caregivers, such as patient family member(s), that might be with the patient to support additional services, such buying of medicine, providing meals to the patient, and other important services which the patient needs. Therefore, caregivers or family members of a patient can access the information of his/her patient via cellular phone through a medical application installed. This is also considered significant to inform the caregiver in case of an emergency or an indication for patient services, for that they are responsible (like patients' medicine intake schedule, eating, and others, while he/she is not near to the patient, or outside the patient room or medical ward.

3.4. Chat Engine and Special Purpose Access. Chat engine facility, in Figure 5, is also a part of proposed system design which was employed to provide group chat service (or text communication) among the medical advisors appointed or working in the medical center. Through several discussions made in a group, the chat would be useful to fight against the critical diseases and to make the patient's health with more care.

The proposed system is deployed using private computing platform and is fully accessible to retrieve the overall information that only is limited to the area of medical center; however, in few critical cases, the information will also be retrievable via the cellular devices, outside of medical center boundary, or everywhere, registered under the names of medical advisors. So, using this facility, the medical advisors are allowed to view the current medical status of their designated patients continuously, even though they are also allowed to send feedback, for example, for health treatments, through the use of cellular application chat box, via the Internet. Therefore, these are significant demonstrations to avoid some major medical issues happening with the patients.

3.5. Information Capturing and Storage. In studies [31-36], developments have been made to retrieve patient's realtime medical information or medical status, over the far distance, or remotely from everywhere, which were significant in getting of information and in monitoring, as well. But in the current study based on some requirements, specifically due to the security issues, communication is limited to the local private body area networks (PHBAN), a network which retrieves the information from the patient through employing of medical noninvasive equipment or noninvasive devices attached with the patients; in our case, we use the ECG devices and also ECG belt with wireless sensor (ECGBWS) according to the scenarios, such as inward monitoring (IWM) and outward monitoring. Whereas, this study also deployed a potential security mechanism, through cryptography $[37,38]$, for ECGBWS protection against the unauthorized entities, and would employ the self-launched attacking scenarios that a measurement will be efficient against the insecurities and for measurement computed from security mechanism evaluation (process).

3.5.1. Inward Monitoring (IWM). Inward monitoring (IWM) has been a common development in telemonitoring systems $[6,13]$, which provides monitoring facility to monitor the health of those patients residing in the medical units or 


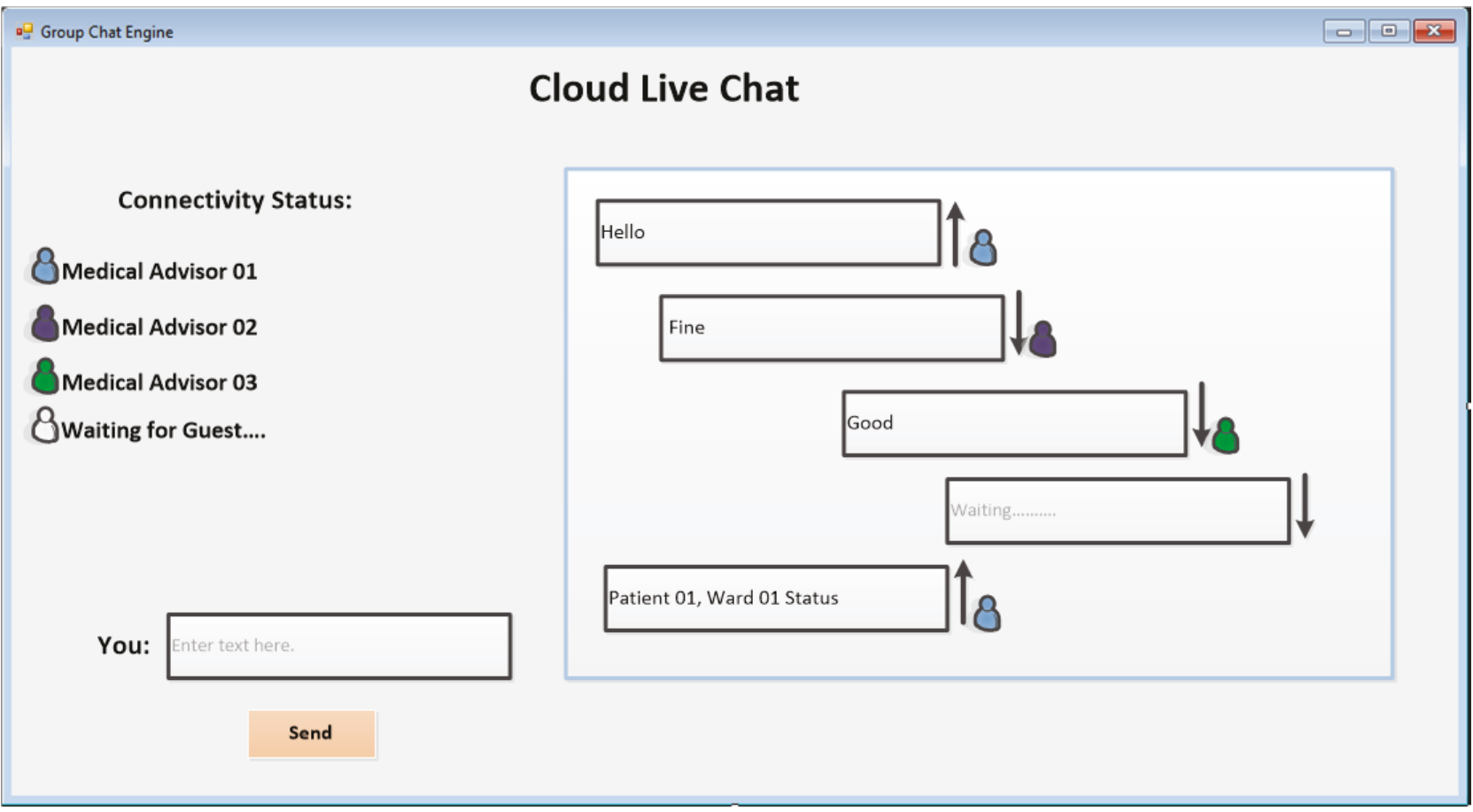

FIGURE 5: Cloud computing: group chat engine.

medical wards; generally, our study case is the same as those existing studies in the area of health monitoring of patient especially for arrhythmia patient. Furthermore, however, we have introduced an application design that was based on simulation, for an instance, is efficient in case the transmission from cloud computing server is disconnected due to system maintenance reasons and also in case if an unauthorized event will occur and measure the overall cloud health computing system.

In real-time monitoring, in case of inward monitoring which covers the area (e.g., width $\times$ height), the information is a continuous measuring from ECG device which attached with the selective parts of the patient body, and the 12 leads are used in our case for arrhythmia measurements. Nowadays, ECG devices are also available that have integration with wireless circuitry, designed to transmit the observed information of arrhythmia patients directly towards the system that has a configuration. For inward monitoring, in case of rhythmic patient's health information measurement and monitoring, the wired network is much better as all the patients (i.e., 10 patients) reside in one medical ward and there are only 10 main LAN cable networks to carry the real-time medical information of each patient in. Nowadays, the ECG devices are equipped with a wired and wireless connectivity; therefore, in Figure 6, to avoid the network's issues as the retrieving of patient's information is critical, the proposed study uses wired LAN connections and as well as wireless connections which are also beneficial in case of disconnection issues relevant to wired networks. Furthermore, some ECG devices are designed with built-in memory that has limited space to store medical information of patient's rhythms and should transfer to external devices through the USB port.
In one medical ward, the numbers of the patients are limited up to 10 patients, in our case of study, that much scalable to accommodate the number of patients (equal to 10); however, these numbers can be increased according to the requirements and based upon the space available. Eventually, through this scenario, several medical wards would be established based on the coming numbers of the patients in the medical center. This study database design is dynamic and would able to store and manage thousands of patient's records, in terms of their personal records and the information that will be measured from ECG devices. In detail, we entered 200 arrhythmia patients in the designed database and were also able to manage their medical information and the corresponding reports upon individual requests (by patients, medical term, and medical advisors). A deemed medical center is designed to accommodate 500 patients' based on the available space or number of built medical units.

In the medical ward, each patient resided in is accommodated with an ECG device attached to measure the continuous electrical signals or events of patients' arrhythmias, through attached leads. Overall, computing measurements, ECG waveforms that show the contractile actions of heart myocardium continuously, are observed and simultaneously displayed on embedded ECG display mainly characterized by three waves, called P, QRS, and T. In these waveforms, the QRS complex is the most important one designated to show the rhythmic activities where $R$ is calculated and represents the peak of QRS complex. ECG is costless and an efficient noninvasive too, for diagnoses of rhythmic diseases and the continuous monitoring of the normal heart activities, in case of irregular activities or waveform intervals, changing measure in amplitude, normal time intervals, and certainly changing in heart patterns (or rhythms shapes) [2]. Further, 

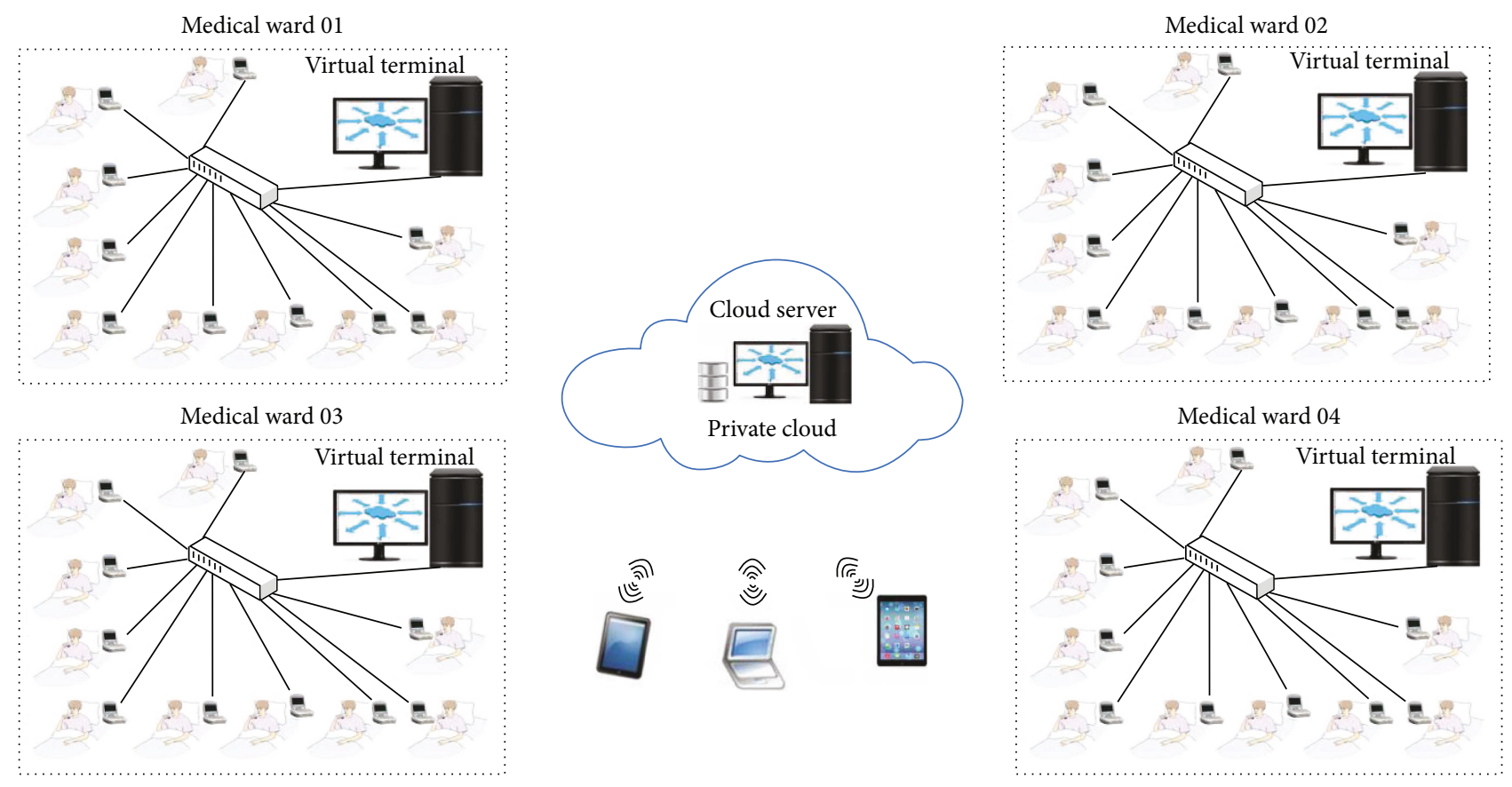

Figure 6: Inward monitoring.

the observed continuous activities of the heart, or QRS complex, are transmitted to the local unit resided in the medical ward, which means that this networked local unit is just a computer system which captures, records, and monitors the real-time rhythms of the patient illustrated in Figure 7, up to 10 patients in a medical ward, through the medical application running from the cloud server. This study is more efficient in computing of designated patients' heart health and would record this information simultaneously back to the server, without any delays of hours, days, and weeks. In Holter monitoring system, rhythms through ECG device were measured and stored in the locally located system then transmitted back after a specified session, and classification on observed medical information was performed offline, which was one of the important limitations of Holter monitoring system [21]. However, in this study, the continuous medical information of patients is captured through the ECG devices, recorded, and monitored in real-time manners, to avoid that any critical issue might happen; further analysis is performed also online through classification and analysis algorithm or application, running from cloud or cloud server. In short, the medical information will be received at the cloud server, from each patient with his/her unique identifier, the diagnostic will be performed based upon the classification algorithm implemented as part of proposed study, and simultaneously, feedback will be transmitted to the designated medical staff and also to the designated advisor for overview and for suggestions in case they will require any. The analysis was always performed based on the static computation of ECG classification algorithm installed and resided as a part of the cloud server.

(1) Application Design and Implementation. Cloud computing is an efficient, reliable, and scalable way for today's application access, more especially in telemonitoring where the medical application, such as ECG information, can retrieve or access and is hosted to monitor and analyze the retrieved medical information through analysis tools, as soft-as-a-service. Mainly, each type of application or software is hosted and can retrieve through the web-based service, where the end-users can simply request and get the desired information, such as ECG/medical information uploaded/ downloaded, medical information analyses, and current and existing reports, according to their queries and demands without the knowledge of application of the underlying complexities and difficulties that might be present in the development or in hosting of any application in cloud computing platform. At a lower layer, in the top-down scenario, cloud service, platform-as-a-service, is employed to manage the processing of the application or software hosted at SaaS layer.

(2) Direct and Offline System. As explained, overall information carried from each patient connected with ECG device is transmitted to a local station located as a part of a medical ward to store and monitor the information in real-time manners through the medical application or virtual machine, running from a cloud server, which means that carried information is directly stored in the cloud server via local system and monitoring is also performed in real time to avoid the critical cases to happen. At the same time, the proposed system design is also efficient to store the medical information in case of system disconnectivity, system connection failure, or other network or system internal issues; so if these cases might happen, then the offline system would be functional. In the offline system, the medical running application is designed with efficiency to receive the upcoming medical information from each patient and store it into a local memory that configures as a part of application software. While 


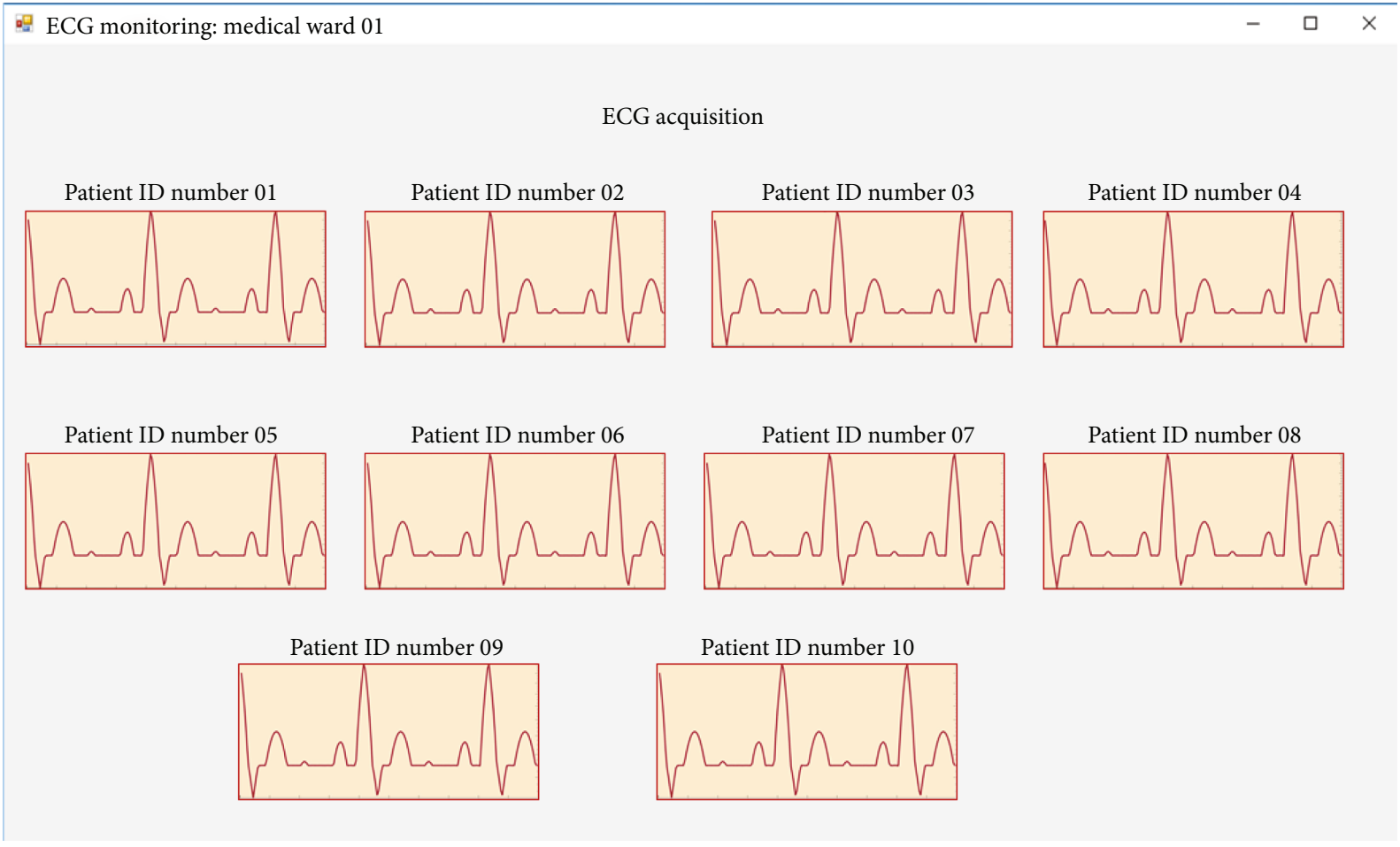

FIGURE 7: ECG inward monitoring.

server connection will again establish, all stored medical information will be updated in the server site database. The offline system scenario is significant in case to handle the future system failures or disconnectivity issues; also, the analysis will be performed in cases of cloud server connectivity issues. To make an analysis, a replicate copy of ECG classification algorithm is always a part of the local system, in each medical ward, that will be activated only when the cloud server will disconnect. As the server will not be in connection anymore, offline bytes or system disconnection bytes are constantly received as a part of medical application, running in the local system, within 20-40 seconds interval and the application will activate the ECG classification algorithm. As consequence, the upcoming ECG information will be stored and at the same time, the medical analysis will be performed locally in each medical ward, and the medical information will be stored and analyzed to fight against the potential critical medical events of arrhythmia.

3.5.2. Outward Monitoring (OWM). In the second phase of the proposed study, outward monitoring (OWM) is modeled to monitor the medical status of rhythm patients, while they are out from their medical wards where they most of time resided for medical treatments and also for other purposes. In most common cases, medical hardware is an equipment in different rooms within one building block or in another block, in case the medical center is surrounded by many blocks, such as blocks A, B, and C. Therefore, the movements of patients are always carried out during their medical tests, heart operations, and heart surgery and in cases of recovery corresponding with required exercises and also in cases of open-air relaxation and spending of good time of gossip with family members and friends. In short, there are many situations and medical reasons in which patients have required for moving out of their medical ward. Therefore, during that time, measuring the rhythms of the patient will be required to avoid the critical cases of arrhythmia while they are out of their medical wards. Eventually, this study proposed and modeled a sensor-based wireless network scenario to capture the ECG waveforms of patients that might be moving out of their medical ward, without connectivity with ECG devices, through the wireless ECG belt, embedded ECG sensor, fixed over the patient body.

While the patient is outside of his/her medical ward, a belt is fixed over the specific part of the patient body that has embedded ECG sensor, having a unique entity, communicating with the cloud server through the various access points (APs). A medical wireless sensor network is designed, in which sensors (or access points) are placed or networked in an area that covered most parts of the medical center. Therefore, real-time medical information could be accessed through wireless sensor configuration, and patient will be located anywhere in the boundary of the medical center or in coverage of wireless sensor network. In Figure 8, wireless sensor network is designed to carry the transmission of medical information from patients, located outside of their medical wards, through ECG sensor belt.

\section{Results and Discussion}

This study is based on the simulation design in which inward and outward networks were modeled to carry the medical information of arrhythmia patients in real-time fashion. As the medical information is important, therefore, it has to be 


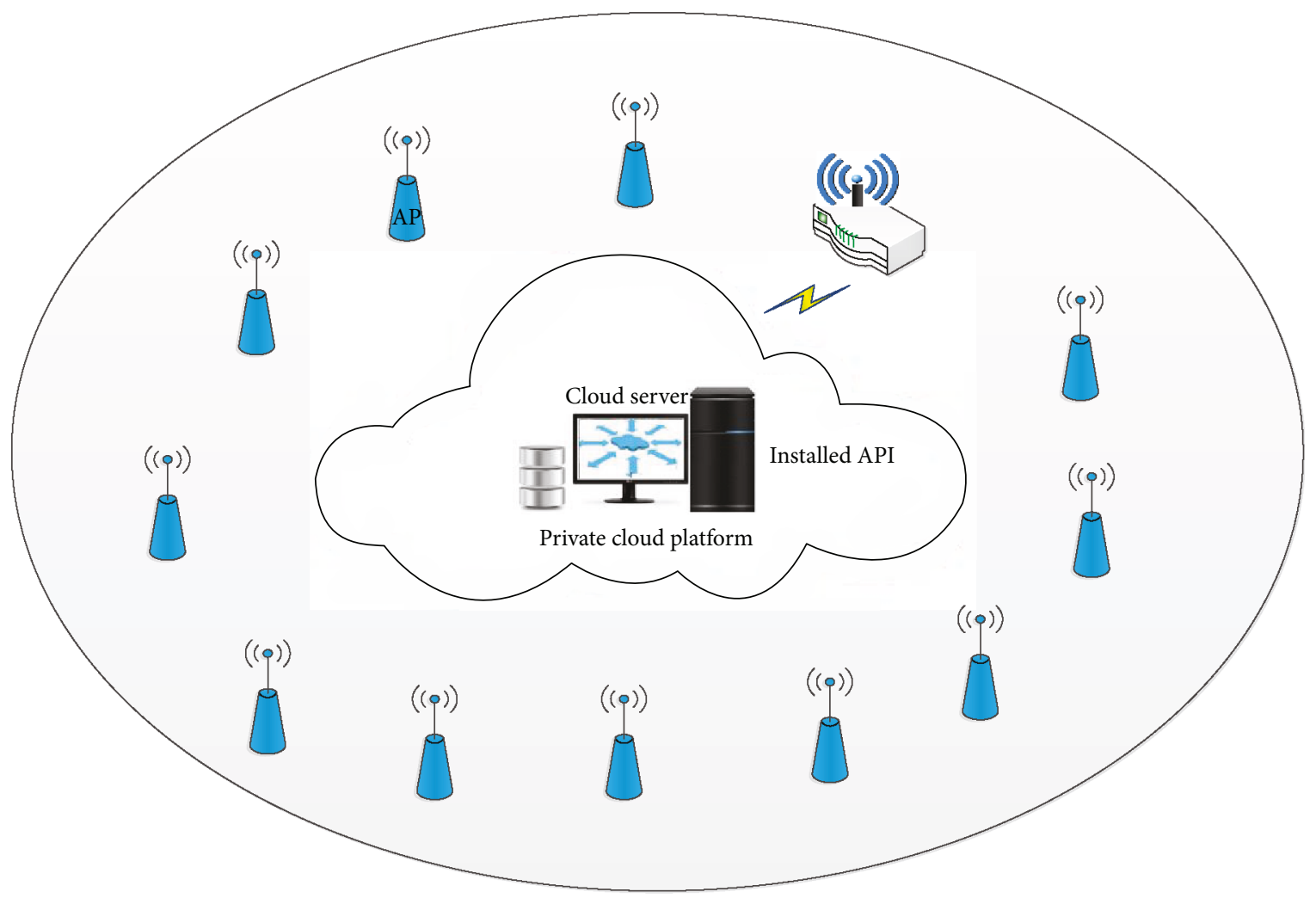

FIGURE 8: ECG outward monitoring.

measured continuously and in real-time manners. Overall, cloud computing designed for accessing, monitoring, and storing of arrhythmia patients' information is simulated in a desktop of a computer system, with system specifications of (1) Intel ${ }^{\circledR}$ Core $^{\mathrm{TM}}$ i7-4790 CPU at a $3.60 \mathrm{GHz}$ processor, (2) $8.00 \mathrm{~GB}$ installed memory, (3) 64-bit operating system, Windows 10, ×64-based processor, (4) Realtek PCIe GBE Family Controller, Ethernet, (5) 1000/1000 (Mbps) link speed, (6) IPv6, and others. A total of six virtual machines were deployed in private cloud system, four were installed to carry and manage the medical information coming from the medical wards (i.e., four medical wards), and the remaining virtual machines such as cloud VM number 05 and cloud VM number 06 were deployed for patient registration and administration purposes and for monitoring the medical information from ECG belt, while patients are in outward monitoring mode, through API installed in VM number 06 in private cloud.

In Table 1, the performance results are measured, in which ECG information was accessed and stored in cloud storage (i.e., in MySQL database tool), in case of inward monitoring and outward monitoring modes. A total of 50 experiments were performed to measure the results, such as ECG signals or information received and stored at the cloud server and security computations, while experiment number 00 is not mentioned in Table 1 but was performed to test the overall cloud system configuration in order to avoid the transmission errors. Each time ECG signals are received at the cloud server, the security was tested to ensure the authentication and confidentiality of that information through employing of AES cryptography algorithm $[37,38]$. Therefore, the signs, such as $\sqrt{ }$ and $X$, are used to show that the ECG information was received and the security test was verified or not. As a consequence, the performance results shown in Table 1 are significant, as high accuracy is computed based on 50 experiment results.

\section{Conclusion and Future Work}

As the technological demands are increasing, the medical systems have been updated with the new advanced technologies (i.e., smart medical systems); however, there are still several challenges, such as to carry the remotely located information and the remote connectivity issues, mainly present in the healthcare systems. Alongside with this, the cost is also a big issue, raising while updating the existing systems with the new developments. Therefore, the cloud computing platform is one of the best solutions in the current age to fight against the issues. In this study, based on the medical organizational specifications and requirements for communication, including the security requirements, private cloud computing environment is designed and modeled where the medical information of heart patients who underregistered or resided in the hospital, inside of the medical ward and outside of the medical ward in the premises of the hospital, is accessed and monitored in real-time manners. Further, the system's security requirements are fulfilled through the implementation of AES algorithm, which resulted in protection of overall communication in the designed private cloud computing environment. Moreover, the study provided an immense 
TABle 1: Performance results.

\begin{tabular}{|c|c|c|c|c|c|}
\hline \multirow{2}{*}{ Number } & \multicolumn{2}{|c|}{ Inward monitoring } & \multicolumn{2}{|c|}{ Outward monitoring } & \multirow{2}{*}{ Ward and patient IDs } \\
\hline & ECG signal & Security test & ECG signal & Security test & \\
\hline 1 & $\checkmark$ & $\checkmark$ & $\checkmark$ & $\checkmark$ & Ward number 01 , patient number 01 \\
\hline 2 & $\checkmark$ & $\checkmark$ & $\mathrm{x}$ & $\mathrm{x}$ & Ward number 01 , patient number 02 \\
\hline 3 & $\checkmark$ & $\checkmark$ & $\checkmark$ & $\checkmark$ & Ward number 01 , patient number 03 \\
\hline 4 & $\checkmark$ & $\checkmark$ & $\checkmark$ & $\checkmark$ & Ward number 01 , patient number 04 \\
\hline 5 & $\checkmark$ & $\checkmark$ & $\checkmark$ & $\checkmark$ & Ward number 01 , patient number 05 \\
\hline 6 & $\checkmark$ & $\checkmark$ & $\checkmark$ & $\checkmark$ & Ward number 01 , patient number 06 \\
\hline 7 & $\checkmark$ & $\mathrm{x}$ & $\checkmark$ & $\checkmark$ & Ward number 01 , patient number 07 \\
\hline 8 & $\checkmark$ & $\checkmark$ & $\mathrm{x}$ & $\mathrm{x}$ & Ward number 01 , patient number 08 \\
\hline 9 & $\checkmark$ & $\checkmark$ & $\checkmark$ & $\checkmark$ & Ward number 01, patient number 09 \\
\hline 10 & $\checkmark$ & $\checkmark$ & $\checkmark$ & $\checkmark$ & Ward number 01 , patient number 10 \\
\hline 11 & $\checkmark$ & $\checkmark$ & $\checkmark$ & $\checkmark$ & Ward number 02 , patient number 01 \\
\hline 12 & $\checkmark$ & $\checkmark$ & $\checkmark$ & $\checkmark$ & Ward number 02 , patient number 02 \\
\hline 13 & $\checkmark$ & $\checkmark$ & $\checkmark$ & $\checkmark$ & Ward number 02 , patient number 03 \\
\hline 14 & $\checkmark$ & $\checkmark$ & $\checkmark$ & $\checkmark$ & Ward number 02 , patient number 04 \\
\hline 15 & $\checkmark$ & $\checkmark$ & $\checkmark$ & $\checkmark$ & Ward number 02 , patient number 05 \\
\hline 16 & $\checkmark$ & $\checkmark$ & $\checkmark$ & $\checkmark$ & Ward number 02 , patient number 06 \\
\hline 17 & $\checkmark$ & $\checkmark$ & $\checkmark$ & $\checkmark$ & Ward number 02, patient number 07 \\
\hline 18 & $\checkmark$ & $\checkmark$ & $\mathrm{x}$ & $\mathrm{x}$ & Ward number 02 , patient number 08 \\
\hline 19 & $\mathrm{x}$ & $\mathrm{x}$ & $\checkmark$ & $\checkmark$ & Ward number 02 , patient number 09 \\
\hline 20 & $\checkmark$ & $\checkmark$ & $\checkmark$ & $\checkmark$ & Ward number 02 , patient number 10 \\
\hline 21 & $\checkmark$ & $\checkmark$ & $\checkmark$ & $\checkmark$ & Ward number 03 , patient number 01 \\
\hline 22 & $\checkmark$ & $\checkmark$ & $\checkmark$ & $\checkmark$ & Ward number 03 , patient number 02 \\
\hline 23 & $\checkmark$ & $\checkmark$ & $\checkmark$ & $\checkmark$ & Ward number 03 , patient number 03 \\
\hline 24 & $\checkmark$ & $\checkmark$ & $\checkmark$ & $\checkmark$ & Ward number 03 , patient number 04 \\
\hline 25 & $\checkmark$ & $\checkmark$ & $\checkmark$ & $\checkmark$ & Ward number 03 , patient number 05 \\
\hline 26 & $\checkmark$ & $\checkmark$ & $\checkmark$ & $\checkmark$ & Ward number 03 , patient number 06 \\
\hline 27 & $\checkmark$ & $\checkmark$ & $\checkmark$ & $\checkmark$ & Ward number 03 , patient number 07 \\
\hline 28 & $\checkmark$ & $\checkmark$ & $\checkmark$ & $\checkmark$ & Ward number 03 , patient number 08 \\
\hline 29 & $\checkmark$ & $\checkmark$ & $\checkmark$ & $\checkmark$ & Ward number 03, patient number 09 \\
\hline 30 & $\checkmark$ & $\checkmark$ & $\checkmark$ & $\checkmark$ & Ward number 03 , patient number 10 \\
\hline 31 & $\checkmark$ & $\checkmark$ & $\checkmark$ & $\checkmark$ & Ward number 04 , patient number 01 \\
\hline 32 & $\checkmark$ & $\checkmark$ & $\checkmark$ & $\checkmark$ & Ward number 04 , patient number 02 \\
\hline 33 & $\checkmark$ & $\checkmark$ & $\mathrm{x}$ & $\mathrm{x}$ & Ward number 04 , patient number 03 \\
\hline 34 & $\checkmark$ & $\checkmark$ & $\checkmark$ & $\checkmark$ & Ward number 04 , patient number 04 \\
\hline 35 & $\checkmark$ & $\mathrm{x}$ & $\checkmark$ & $\checkmark$ & Ward number 04 , patient number 05 \\
\hline 36 & $\checkmark$ & $\checkmark$ & $\checkmark$ & $\checkmark$ & Ward number 04, patient number 06 \\
\hline 37 & $\checkmark$ & $\checkmark$ & $\checkmark$ & $\checkmark$ & Ward number 04 , patient number 07 \\
\hline 38 & $\checkmark$ & $\checkmark$ & $\checkmark$ & $\checkmark$ & Ward number 04 , patient number 08 \\
\hline 39 & $\checkmark$ & $\checkmark$ & $\checkmark$ & $\checkmark$ & Ward number 04 , patient number 09 \\
\hline 40 & $\checkmark$ & $\checkmark$ & $\checkmark$ & $\checkmark$ & Ward number 04 , patient number 10 \\
\hline 41 & $\checkmark$ & $\checkmark$ & $\checkmark$ & $\checkmark$ & Ward number 01 , patient number 03 \\
\hline 42 & $\checkmark$ & $\checkmark$ & $\checkmark$ & $\checkmark$ & Ward number 01 , patient number 06 \\
\hline 43 & $\checkmark$ & $\checkmark$ & $\checkmark$ & $\checkmark$ & Ward number 02 , patient number 02 \\
\hline 44 & $\checkmark$ & $\checkmark$ & $\checkmark$ & $\checkmark$ & Ward number 03 , patient number 03 \\
\hline 45 & $\checkmark$ & $\checkmark$ & $\checkmark$ & $\checkmark$ & Ward number 04 , patient number 01 \\
\hline 46 & $\checkmark$ & $\checkmark$ & $\checkmark$ & $\checkmark$ & Ward number 03 , patient number 01 \\
\hline 47 & $\checkmark$ & $\checkmark$ & $\checkmark$ & $\checkmark$ & Ward number 02 , patient number 05 \\
\hline
\end{tabular}


TABLe 1: Continued.

\begin{tabular}{cccccc}
\hline \multirow{2}{*}{ Number } & \multicolumn{2}{c}{ Inward monitoring } & \multicolumn{2}{c}{ Outward monitoring } & \multirow{2}{*}{ Ward and patient IDs } \\
& ECG signal & Security test & ECG signal & Security test & Ward number 01, patient number 07 \\
\hline 48 & $\checkmark$ & $\checkmark$ & $\checkmark$ & $\checkmark$ & Ward number 03, patient number 09 \\
49 & $\checkmark$ & $\checkmark$ & $\checkmark$ & $\checkmark$ & Ward number 01, patient number 08 \\
50 & $\checkmark$ & $\checkmark$ & $\checkmark$ & $\checkmark$ & \\
\hline
\end{tabular}

platform of knowledge against the causes of arrhythmia and about the arrhythmia chat engine in which medical advisors can share their ideas and suggestions that will be remarkable to fight against the enormous condemnatory heart diseases.

In early future work, this study will be extended with the new developments of hybrid cloud computing, where the main goal is to provide the medical services to the remotely located patients and the concept to measure the medical information continuously. Moreover, the study will be updated with the newly used technology in the arena of information technology called the Internet of things (IoT), which provides automated communication services over the Internet access and will be helpful for the development of new advanced medical systems.

\section{Conflicts of Interest}

The authors declare that there is no conflict of interests regarding the publication of this paper.

\section{Acknowledgments}

This work was supported by the Institute for Information and Communications Technology Promotion (IITP) grant funded by the Korean government (MSIT) (no. 2017-000756, development of interoperability and management technology of IoT system with heterogeneous ID mechanism) and the Faculty Research Fund of Sejong University in year the 2017 .

\section{References}

[1] C. Doukas and I. Maglogiannis, "Bringing IoT and cloud computing towards pervasive healthcare," in 2012 Sixth International Conference on Innovative Mobile and Internet Services in Ubiquitous Computing, pp. 922-926, Palermo, Italy, July 2012.

[2] EMC Corporation, "The private cloud for healthcare enables coordinated patient care," 2010, http://middle-east.emc.com/ collateral/emc-perspective/h7349-hc-private-cloud-ep.pdf.

[3] L. Heilig and S. Voss, "A scientometric analysis of cloud computing literature," IEEE Transactions on Cloud Computing, vol. 2, no. 3, pp. 266-278, 2014.

[4] K. Gai and S. Li, "Towards cloud computing: a literature review on cloud computing and its development trends," in 2012 Fourth International Conference on Multimedia Information Networking and Security, vol. 146, p. 142, Nanjing, China, November 2012.

[5] P. Mell and T. Grance, The NIST Definition of Clouding Computing Recommendations National Inst. of Standards and
Technology, vol. 145, NIST Special Publication, Gaithersburg, MD, USA, 2011.

[6] D. Chappell, "The Microsoft private cloud, a technology overview," 2011, http://www.davidchappell.com/writing/white_ papers/The_Microsoft_Private_Cloud_v1.0-Chappell.pdf.

[7] M. D. Lakshmi and J. P. M. Dhas, "An open source private cloud solution for rural healthcare," in 2011 International Conference on Signal Processing, Communication, Computing and Networking Technologies, pp. 670-674, Thuckafay, India, July 2011.

[8] S. Pandey, W. Voorsluys, S. Niu, A. Khandoker, and R. Buyya, "An autonomic cloud environment for hosting ECG data analysis services," Future Generation Computer Systems, vol. 28, no. 1, pp. 147-154, 2012.

[9] Y. Wei, K. Sukumar, C. Vecchiola, D. Karunamoorthy, and R. Buyya, "Aneka cloud application platform and its integration with windows azure," in Cloud Computing: Methodology, Systems, and Applications, L. Wang, R. Ranjan, J. Chen, and B. Benatallah, Eds., CRC Press, Boca Raton, FL, USA, 2011.

[10] C. Vecchiola, X. Chu, and R. Buyya, "Aneka: a software platform for .NET based cloud computing," High Speed and Large Scale Scientific Computing, vol. 18, pp. 267-295, 2009.

[11] H. Alemdar and C. Ersoy, "Wireless sensor networks for healthcare: a survey," Computer Networks, vol. 54, no. 15, pp. 2688-2710, 2010.

[12] A. Milenković and C. Otto, "Wireless sensor networks for personal health monitoring: issues and an implementation," Computer Communications, vol. 29, no. 13-14, pp. 25212533, 2006.

[13] R. Shahriyar, M. F. Bari, G. Kundu, S. I. Ahamed, and M. M. Akbar, "Intelligent Mobile Health Monitoring System (IMHMS)," in Electronic Healthcare. eHealth 2009, vol. 27 of Lecture Notes of the Institute for Computer Sciences, Social Informatics and Telecommunications Engineering, pp. 5-12, Springer, Berlin, Heidelberg, 2009.

[14] B. E. Reddy, T. V. S. Kumar, and G. Ramu, "An efficient cloud framework for health care monitoring system," in 2012 International Symposium on Cloud and Services Computing, pp. 113-117, Mangalore, India, December 2012.

[15] M. Al-Qurishi, M. Al-Rakhami, F. Al-Qershi et al., "A framework for cloud-based healthcare services to monitor noncommunicable diseases patient," International Journal of Distributed Sensor Networks, vol. 11, no. 3, Article ID 985629, 2015.

[16] J. Mišić and V. Mišić, "Wireless sensor networks: performance, reliability, security, and beyond," Computer Communications, vol. 29, no. 13-14, pp. 2447-2449, 2006.

[17] J. G. Ko, L. Chenyang, M. B. Srivastava, J. A. Stankovic, A. Terzis, and M. Welsh, "Wireless sensor networks for healthcare," Proceedings of the IEEE, vol. 98, no. 11, pp. 1947-1960, 2010.

[18] R. D. Caytiles and S. Park, "A study of the design of wireless medical sensor network based u-Healthcare system," 
International Journal of Bio-Science and Bio-Technology, vol. 6, no. 3, pp. 91-96, 2014.

[19] T. Gao, C. Pesto, L. Selavo et al., "Wireless medical sensor networks in emergency response: implementation and pilot results," in 2008 IEEE Conference on Technologies for Homeland Security, vol. 192, p. 187, Waltham, MA, USA, May 2008.

[20] N. Fernando, S. W. Loke, and W. Rahayu, "Mobile cloud computing: a survey," Future Generation Computer Systems, vol. 29, no. 1, pp. 84-106, 2013.

[21] C. Wen, M.-F. Yeh, K.-C. Chang, and R.-G. Lee, "Real-time ECG telemonitoring system design with mobile phone platform," Measurement, vol. 41, no. 4, pp. 463-470, 2008.

[22] W. Yao, C.-H. Chu, and Z. Li, "The use of RFID in healthcare: benefits and barriers," 2010 IEEE International Conference on RFID-Technology and Applications, vol. 134, 2010, p. 128, 2010.

[23] H. Al Nahas and J. S. Deogun, "Radio frequency identification applications in smart hospitals," in Twentieth IEEE International Symposium on Computer-Based Medical Systems (CBMS'07), vol. 342, p. 337, Maribor, Slovenia, June 2007.

[24] M. Mayer, N. Görtz, and J. Kaitovic, "RFID tag acquisition via compressed sensing," in 2014 IEEE RFID Technology and Applications Conference (RFID-TA), pp. 26-31, Tampere, Finland, September 2014.

[25] C. D. Kidd, R. Orr, G. D. Abowd et al., "The aware home: a living laboratory for ubiquitous computing research," in Cooperative Buildings. Integrating Information, Organizations, and Architecture. CoBuild 1999, N. A. Streitz, J. Siegel, V. Hartkopf, and S. Konomi, Eds., vol. 1670 of Lecture Notes in Computer Science, pp. 191-198, Springer, Berlin, Heidelberg, 1999.

[26] E. A. Fry and L. A. Lenert, "MASCAL: RFID tracking of patients, staff and equipment to enhance hospital response to mass casualty events," AMIA Annual Symposium Proceedings Archive, vol. 2005, pp. 261-265, 2005.

[27] D. I. Tapia, R. S. Alonso, Ó. García, F. de la Prieta, and B. Pérez-Lancho, "Cloud-IO: cloud computing platform for the fast deployment of services over wireless sensor networks," in 7th International Conference on Knowledge Management in Organizations: Service and Cloud Computing, L. Uden, F. Herrera, J. Bajo Pérez, and J. Corchado Rodríguez, Eds., vol. 172 of Advances in Intelligent Systems and Computing, Springer, Berlin, Heidelberg, 2013.

[28] D. I. Tapia, R. S. Alonso, J. F. De Paz, C. Zato, and F. de la Prieta, "A telemonitoring system for healthcare using heterogeneous wireless sensor networks," International Journal of Artificial Intelligence, vol. 6, 2011.

[29] K. Dongkyun, J. Ha, and K. You, "Adaptive extended Kalman filter based geolocation using TDOA/FDOA," International Journal of Control and Automation, vol. 4, no. 2, pp. 49-58, 2010.

[30] H. Liu, H. Darabi, P. Banerjee, and J. Liu, "Survey of wireless indoor positioning techniques and systems," IEEE Transactions on Systems, Man, and Cybernetics, Part C: Applications and Reviews, vol. 37, no. 6, pp. 1067-1080, 2007.

[31] N. Amour, A. Hersi, N. Alajlan, Y. Bazi, and H. AlHichri, Implementation of a Mobile Health System for Monitoring ECG Signals, CiteSeerx, Princeton, NJ, USA, 2015.

[32] R. S. Tolentino, Y.-T. Kim, B. Park, and G.-C. Park, “A Design and Analysis Ubiquitous Healthcare Monitoring System over Wireless Sensor Network," International Journal of Multimedia and Ubiquitous Engineering, vol. 6, 2011http://www.sersc.org.
[33] D. Aranki, G. Kurillo, P. Yan, D. M. Liebovitz, and R. Bajcsy, "Real-time tele-monitoring of patients with chronic heartfailure using a smartphone: lessons learned," IEEE Transactions on Affective Computing, vol. 7, no. 3, pp. 206-219, 2016.

[34] D. Dziak, B. Jachimczyk, and W. J. Kulesza, "IoT-based information system for healthcare application: design methodology approach," Applied Sciences, vol. 7, no. 6, p. 596, 2017.

[35] V. P. Cornet and R. J. Holden, "Systematic review of smartphone-based passive sensing for health and wellbeing," Journal of Biomedical Informatics, vol. 77, pp. 120-132, 2018.

[36] N. Xiong, A. V. Vasilakos, L. T. Yang et al., "Comparative analysis of quality of service and memory usage for adaptive failure detectors in healthcare systems," IEEE Journal on Selected Areas in Communications, vol. 27, no. 4, pp. 495-509, 2009.

[37] A. Shahzad, M. Lee, H. D. Kim, S.-M. Woo, and N. Xiong, "New security development and trends to secure the SCADA sensors automated transmission during critical sessions," Symmetry, vol. 7, no. 4, pp. 1945-1980, 2015.

[38] S. Musa, A. Shahzad, and A. Aborujilah, "Secure security model implementation for security services and related attacks base on end-to-end, application layer and data link layer security," in ICUIMC '13 Proceedings of the 7th International Conference on Ubiquitous Information Management and Communication, p. 8, Kota Kinabalu, Malaysia, January 2013. 


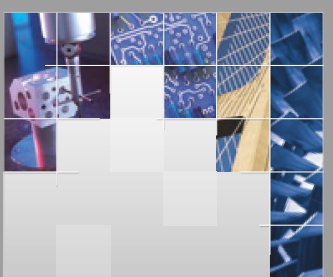

\section{Enfincering}
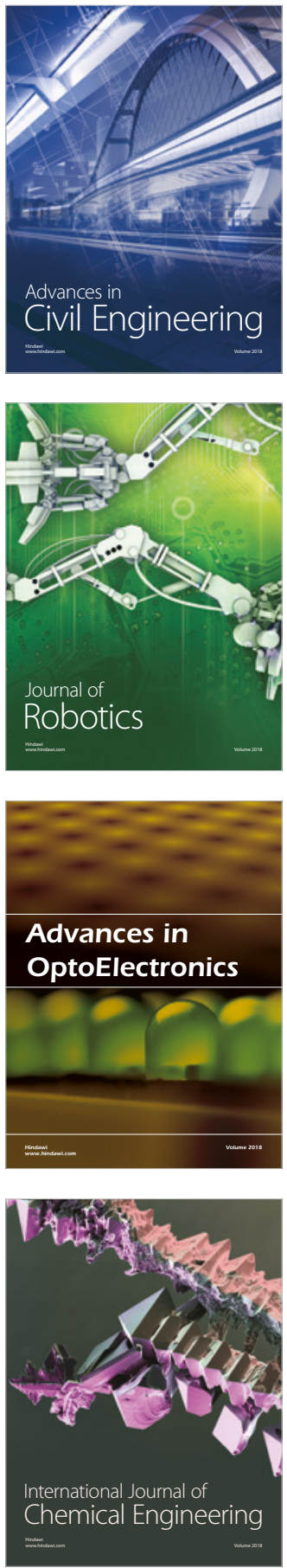

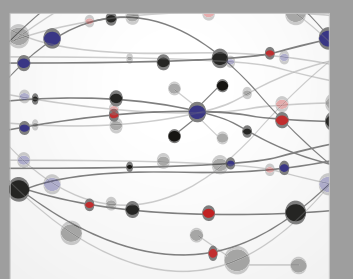

\section{Rotating \\ Machinery}

The Scientific World Journal

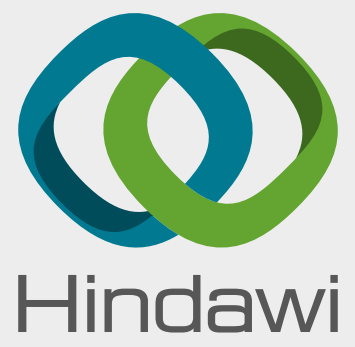

Submit your manuscripts at

www.hindawi.com
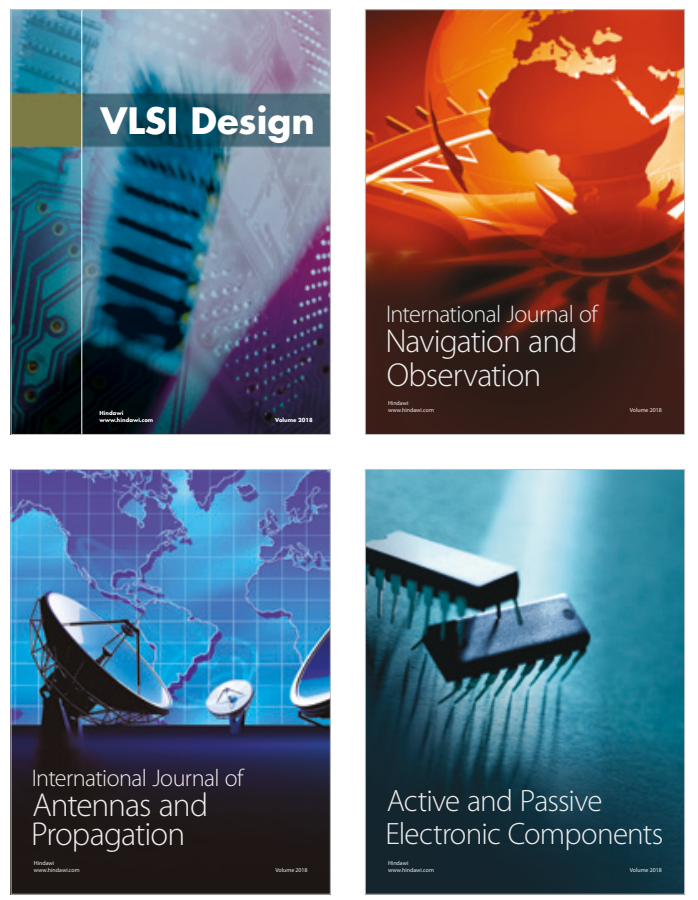
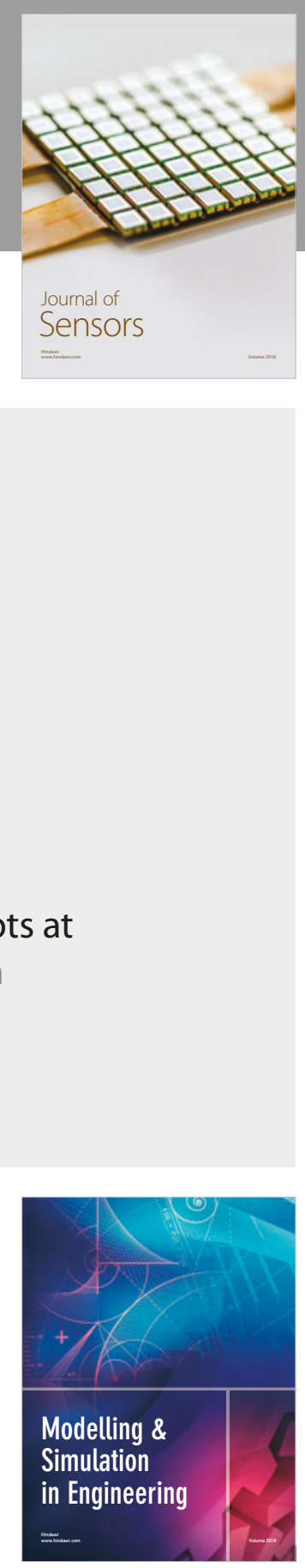

\section{Advances \\ Multimedia}
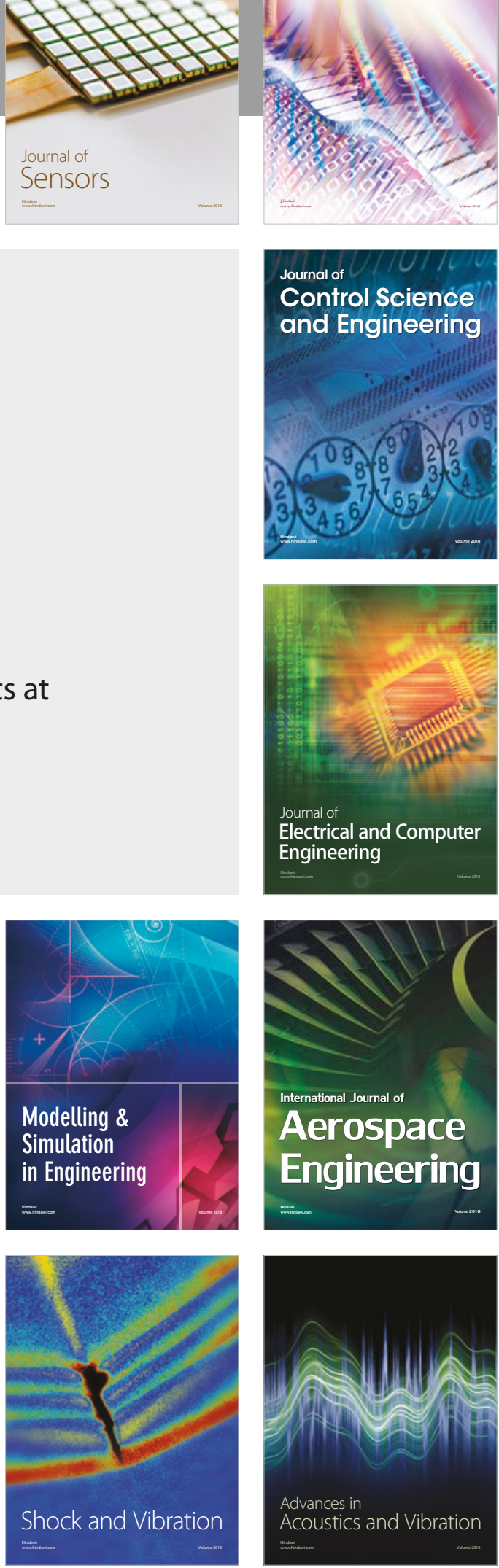